\title{
Quantitative assessment of atherosclerotic lesion of rabbit aorta by compressed slice analysis of magnetic resonance images ex vivo
}

\author{
Byung HS. Cho*1,2, Denis Kholine ${ }^{3}$, Byung H. Chung ${ }^{4}$, Boris Odintsov ${ }^{5}$ \\ ${ }^{1}$ Harlan E. Moore Heart Research Foundation, Champaign, Illinois, USA \\ ${ }^{2}$ Division of Nutritional Sciences, University of Illinois, Urbana, Illinois, USA \\ ${ }^{3}$ Department of Veterinary Clinical Medicine, University of Illinois, Urbana, Illinois, USA \\ ${ }^{4}$ Department of Nutrition Sciences, University of Alabama at Birmingham, Alabama, USA \\ ${ }^{5}$ Biomedical Imaging Center of the Beckman Institute for Advanced Science and Technology, Department of Bioengineering, \\ University of Illinois, Urbana, Illinois, USA
}

Received: August 10, 2015

Accepted: September 13, $2015 \quad$ Online Published: September 29, 2015

DOI: $10.5430 /$ jbgc.v6n1p1

URL: http://dx.doi.org/10.5430/jbgc.v6n1p1

\begin{abstract}
The objective of the present study was to evaluate a new compressed slice analysis technique of aortas ex vivo. Atherosclerosis was developed in rabbits by feeding an atherogenic diet containing $2 \%$ cholesterol and $6 \%$ peanut oil for 8 weeks. At termination, aortas were excised and imaged ex vivo by Magnetic resonance images (MRI). MRI data were acquired on a super high magnetic field vertical bore imaging scanner equipped with a Unity/Inova console, operating at $14.1 \mathrm{~T}$ and dedicated to small animal studies. $\mathrm{T}_{2}$-weighted coronal 2-D sections were acquired first to determine a ROI (region of interest) of the thoracic aorta using a Spin-Echo multi slice pulse sequence. Then, $\mathrm{T}_{2}$-weighted images were acquired in transversal sections of ROI slice by slice $(2 \mathrm{~mm} / \mathrm{slice})$ in the total length of $4 \mathrm{~cm}$. The MRI images of 20 slices were then compressed into a single image, and the resultant compressed single image was used as a quantitative characteristic of atherosclerotic lesions. By utilizing the compressed slices (CS) analysis, the imaging intensity of atherosclerotic lesions was calculated. A significant correlation was found between the quantitative CS-MRI and the aortic cholesterol content (Pearson correlation coefficient $r=0.95$ ). The present findings demonstrate that the compressed slice analysis technique of MRI images could be applied for a quantitative assessment of the progression and/or regression of atherosclerosis.
\end{abstract}

Key Words: Atherosclerosis, Cholesterol, Compression, MRI images

\section{INTRODUCTION}

Atherosclerotic disease is one of the leading causes of morbidity and mortality. ${ }^{[1,2]}$ Numerous non-invasive tests have been developed in order to quantify atherosclerotic lesions in humans and animals with arterial disease. Considerable progress has been achieved by ultrasound technologies al- lowing visualization. ${ }^{[3]}$ On the other hand, in non-accessible areas, such as the abdominal aorta and renal arteries the use of this technique is still in progress. ${ }^{[4]}$ Tearney et al..$^{[5]}$ applied optical coherence tomography to quantify macrophage content in atherosclerotic lesions. The Magnetic Resonance Imaging (MRI) methodology has received extensive evalua-

*Correspondence: Byung HS. Cho; Email: bhcho@illinois.edu; Address: Harlan E. Moore Heart Research Foundation, Champaign, Illinois, USA. 
tion in recent years. MRI of atherosclerotic lesions was initially demonstrated ex vivo with the use of both imaging and spectroscopic methods. ${ }^{[6,7]}$ In vivo lesions imaging has been more challenging, given the presence of biological motion and time constraints. There are several known difficulties with the characteristics of the MRI data related to structural and chemical heterogeneities of the atherosclerotic lesions in vessels, which result in a complex MRI vessel-wall signal. The most common approach to the atherosclerotic lesions analysis is based on geometrical matching MRI results with corresponding histopathological aorta lesion areas. MRI was also applied successfully to experimental in vivo studies in which the progression/regression of atherosclerotic lesions could be established. ${ }^{[8,9]}$ Skinner et al. ${ }^{[10]}$ reported MRI of lesions progression in 6 balloon-injured rabbits placed on a high-cholesterol diet for up to 16 months. Recent findings suggest that a non-invasive magnetic resonance imaging technology may provide significant help identifying the histopathologic nature of the lesions. Helft et al. ${ }^{[11]}$ also investigated the in vivo noninvasive MRI to quantify fibrotic and lipid components of atherosclerosis in rabbits.

In the present study, a compression technique of multi-MRI slice images into a single image was developed, and the resultant compressed single image was used as a quantitative characteristic of atherosclerotic lesions. Utilizing the Compressed Slices Histogram (CSH) analysis developed, the imaging intensity of atherosclerotic lesions was then calculated. Since the accumulation of cholesterol is a characteristic feature of atherosclerosis, the cholesterol content of the imaged section of thoracic aorta was also measured. A significant correlation was found between the quantitative CS-MRI and the aortic cholesterol content (Pearson correlation coefficient $r=0.95$ ).

\section{Methods}

\subsection{Animals and diet}

Eight New Zealand White male rabbits (Myrtle's Rabbitry), 8-weeks old weighing 4-5 lbs, were housed in stain-less steel cages, and experiments were performed with the approval of the University of Illinois Institutional Animal Care and Use Committee. To induce the development of atherosclerosis, animals were fed ad libitum an atherogenic diet containing $2 \%$ cholesterol and 6\% peanut oil (Teklad, TD 91314) for 8 weeks, and then the diet was returned to the normal rabbit chow for a period of two months. At the termination, rabbits were euthanized using a dose of Na pentobarbital $(26 \%$ solution) at $1 \mathrm{ml} / \mathrm{kg}$ body weight.

The aortas were dissected, cleaned and photographed by a digital camera for the histological record. Before MRI imaging, the open surface of arterial segments were inspected vi- sually. An extensive atherosclerotic lesions development was noted within the wall without staining, e.g. the yellow-shaded areas corresponding to atherosclerotic lesion development (see white areas in Figure 1). It was shown that formalin fixation alters water proton relaxation times in atherosclerotic vascular samples and can affect MRI data. ${ }^{[12]}$ Therefore, all aorta segments had no formalin fixation and were immediately frozen with phosphate buffered saline solution until MRI is performed.
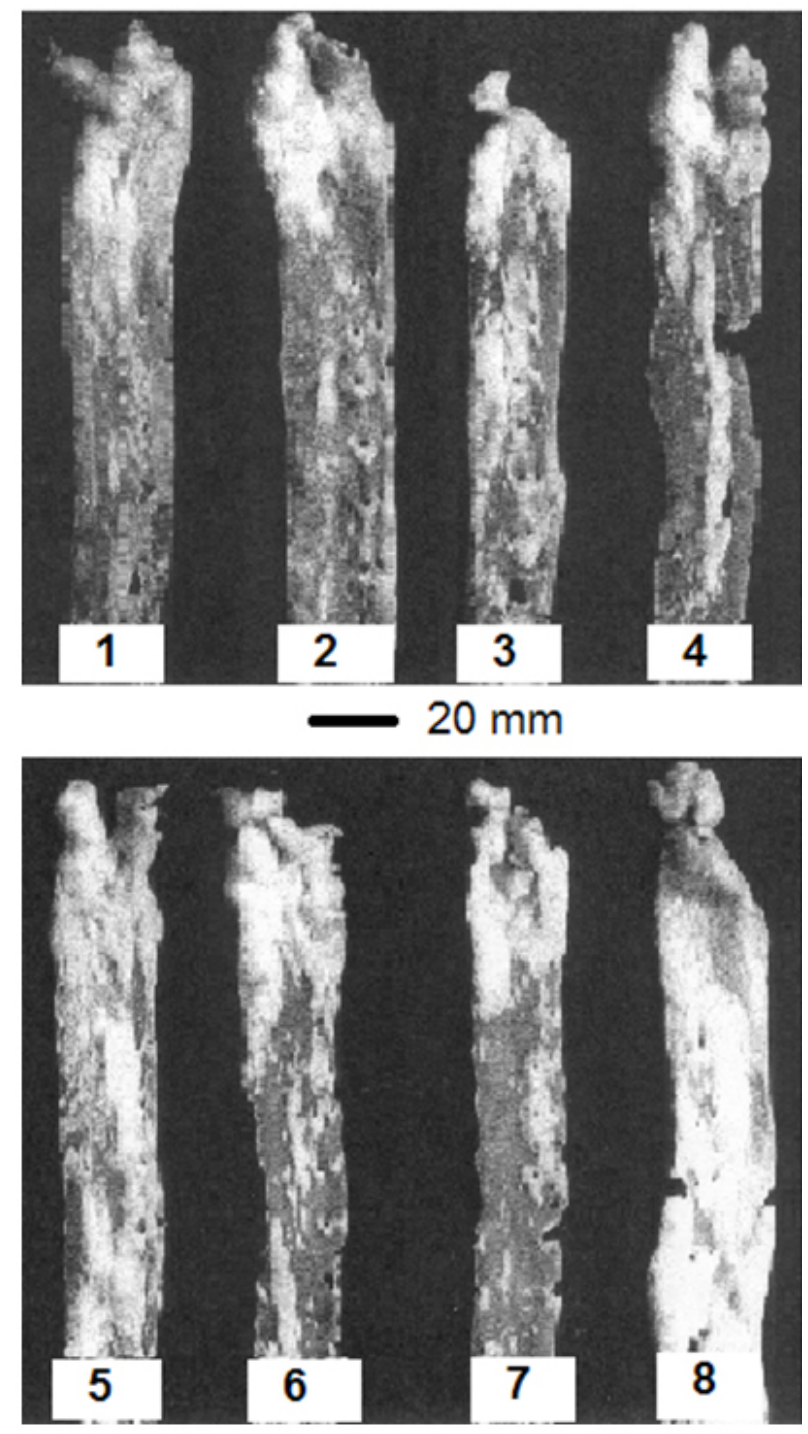

Figure 1. Rabbit aortas with the extensive development of atherosclerosis (white area)

\subsection{MRI}

For MRI measurements the aortas were thawed, and inserted into the glass tubing ( $8 \mathrm{~mm}$ i.d.) in order to mimic the roundform of aortas. The thoracic aorta starting from mediastinal branches to downward $4 \mathrm{~cm}$, which section best-situated inside of the glass tubing, was imaged for atherosclerotic le- 
sions detection and designated as the region of interest (ROI). All aortas were imaged ex vivo at room temperature. Each aorta was placed inside a vertical imaging scanner (Oxford Instruments, Abington, UK) equipped with a Unity/Inova console (Varian/Agilent, Palo Alto, CA), operating at 14.1 $\mathrm{T}$ with a bore of an internal diameter of $5 \mathrm{~cm}$. The gradient coils were driven by a set of Varian amplifiers, creating a maximum gradient of $950 \mathrm{mT} / \mathrm{m}$ at $200 \mathrm{~V}$ and 100 A with a risetime of $15 \mu$ s and a deviation from linearity of less than 5\%. Varian transmitter/receiver quadrature RF coil was used with an internal diameter of $3.0 \mathrm{~cm}$. $\mathrm{T}_{2}$-weighted coronal (along an aorta) 2-D sections were acquired first to determine a region of the most representative aorta lesions formation using a Spin-Echo (SE) multi slice pulse sequence. Figure 2 shows a chosen ROI in aorta with the total $4 \mathrm{~cm}$ length. Then, $\mathrm{T}_{2}$-weighted (SE) images were acquired in transversal sections (see Figure 2a) of chosen ROI in order to obtain a quantitative analysis of the atherosclerotic lesions. The transverse images were acquired slice by slice with no gap between slices using the following set of imaging parameters:

repetition time $(\mathrm{TR})=3,000 \mathrm{~ms}$, echo time $(\mathrm{TE})=30 \mathrm{~ms}$, number of transients /averages $(\mathrm{NT})=1$, spectral width $(\mathrm{SW})=50 \mathrm{kHz}$, field of view $(\mathrm{FOV})=0.8 \times 0.8 \mathrm{~cm}^{2}$, matrix size $=256 \times 256$, in-plain resolution $=30 \times 30 \mu \mathrm{m}^{2}$, slice thickness $=2.0 \mathrm{~mm}$, number of slices $=20$, acquisition time $=10 \mathrm{~min}$. Spin-spin relaxation time $\mathrm{T}_{2}$ value (30 ms) was adjusted experimentally to optimize both the best image quality and $\mathrm{T}_{2}$ contrast of atherosclerotic lesion components.

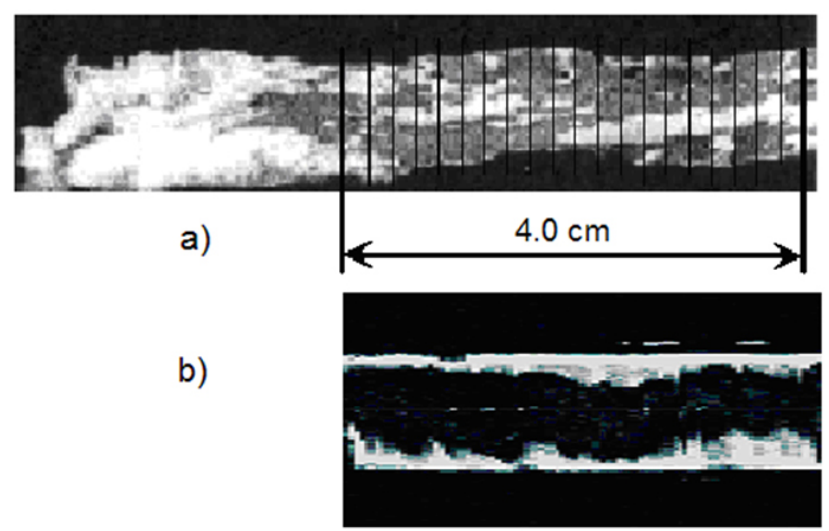

Figure 2. a) The region of the thoracic aorta for MRI imaging; b) $\mathrm{T}_{2}$-weighted ROI coronal $2 \mathrm{D}$ aorta image in the length of $4 \mathrm{~cm}$

\subsection{Cholesterol analysis}

The imaged region $(4 \mathrm{~cm})$ of the thoracic aorta was cut, weighed and homogenized, and the total lipids were extracted with chloroform/methanol $(2: 1, \mathrm{v} / \mathrm{v})$ according to the method of Folch et al. ${ }^{[13]}$ The total lipid extracts were finally dissolved in isopropyl alcohol containing $100 \mathrm{~g}$ Triton $\mathrm{X}-100 / \mathrm{L}{ }^{[14]}$ The total cholesterol concentration was measured calorimetrically using Wako cholesterol-E kits.

\section{RESULTS AND DISCUSSION}

Before MRI imaging, the excised aortas were inspected visually and were photographed by a digital camera for the histological record (see Figure 1). An extensive development of atherosclerosis was noted without staining, e.g. the yellow-shaded areas corresponding to atherosclerotic lesions formation (white areas in Figure 1). Figure 1 also shows the heterogeneous distribution of the atherosclerotic lesions along the aortic wall.

The CSH MRI analysis and fast postprocessing software algorithm was carried out following a flow chart as shown in Figure 3. All original MRI images were stored as FID files and then analyzed with specially designed software utilizing Matlab (6.5.0). In order to reduce noise effects, the signal from the empty (no subject) RF coil was acquired with the same set of MRI acquisition parameters and then subtracted from the aorta compressed image histogram.

Figure 4 shows a typical set of $20 \mathrm{~T}_{2}$ weighted transverse $2 \mathrm{D}$ MRI images in a .jpg (.tiff) format (see step 3 in Figure 3). The set of 20 transverse MRI images has been then summarized (see step 4 in Figure 3) into a single compressed image (see Figure 5).

The 2-dimensional MRI signal acquisition with no gap between slices contains detailed information about atherosclerotic lesions distribution along the aorta. At the same time the subsequent compressed image averages MRI data in a chosen ROI of aorta thus providing statistically weighted results. Figure 6 shows the coordinated system utilized in slice compressing procedure.

To quantify the distribution of the MRI data the histogram of each of the compressed image set was calculated (see Figure 7). Histogram allowed us to determine numerically the weight of intensity for each point value in summary array with number of points (NP) equal to:

$$
N P=n \text { Slice } \times P E \times R O
$$

where $P E$ is a number of points in a phase encoding direction; $R O$ is a number of points in a read out direction; $n$ Slice is a number of slices.

Figure 7 demonstrates the usability of suggested CSH analysis on a modeling system. Figure 7a shows MRI compressed $2 \mathrm{D}$ images of two phantoms with different (and known) pro- 
ton concentrations and different $\mathrm{T}_{2}$ relaxation times. Following the above algorithm and adjusting set of $\mathrm{T}_{2}$-weighting parameters one can easily separate and analyze corresponding histograms shown in Figure 7b. The results indicate that integral histogram intensities (areas under the curves) cor- relate (with a percentage standard deviation of $\pm 2 \%$ ) with proton concentrations in the phantoms. Note that any desirable histogram can be chosen for analysis thus providing a basis for selective quantitative assessment.
Figure 3. Flow chart of fast post-processing CSH algorithm for quantitative MRI of atherosclerosis
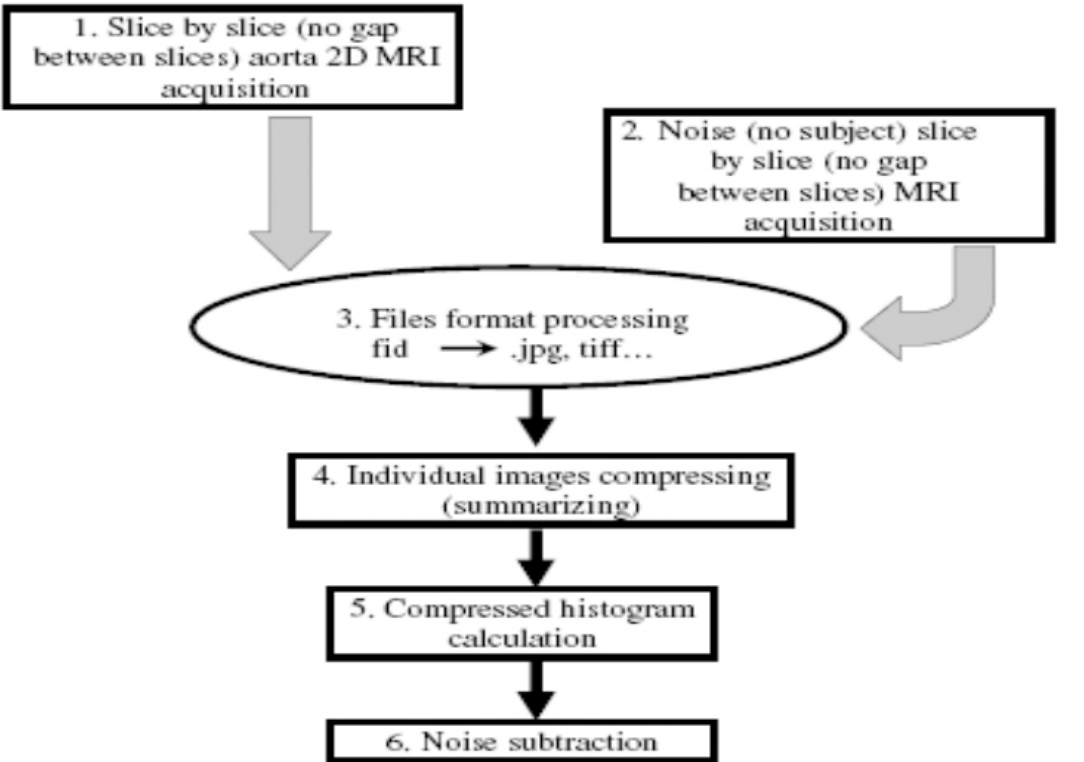

6. Noise subtraction

7. Final histogram integration

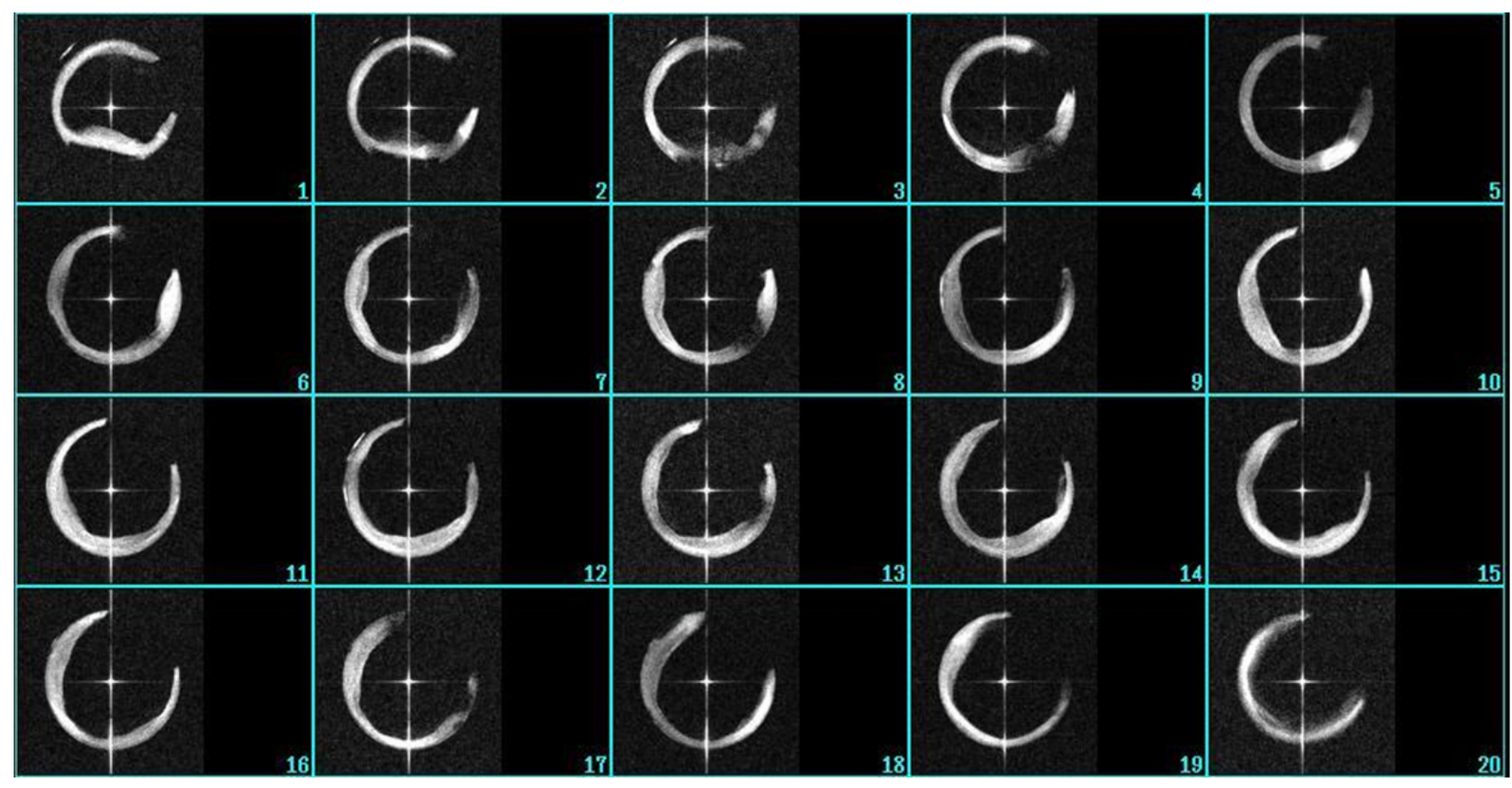

Figure 4. A typical set of 20 slices of rabbit aorta $T_{2}$ weighted transverse 2D images ( $\left.2 \mathrm{~mm} / \mathrm{slice}\right)$ 
$\mathrm{T}_{2}$-weighted imaging has been successfully employed by many researchers for the visualization of fibrous and lipid components of atherosclerotic lesions ${ }^{[15,16]}$ although additional imaging sequences can be applied to assist with the differentiation of all the other components of complex atherosclerotic lesions. ${ }^{[17-19]}$ Utilizing different MRI modalities (e.g. weighting, different imaging pulse sequences, fat suppression, etc.) the developed CSH protocol can be adjusted ("tuned") to contrast any particular atherosclerotic lesion components.

Figure 8 shows integral histogram intensity of the compressed 2D aorta images with a noise subtracted (steps 5-7 in Figure 3). The set of imaging parameters listed in the method section 2 has been employed. Note that spectral filter can be introduced into the processing algorithm in Figure 3 to increase components differentiation of complex atherosclerotic lesions.

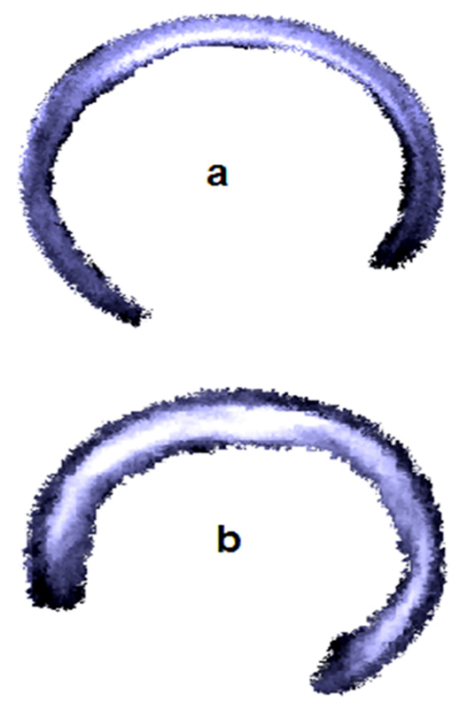

Figure 5. Compressed single transverse $2 \mathrm{D}$ image obtained by summarizing 20 MRI images shown in Figure 4 (a/aorta \#1; b/aorta \#2)

The obtained histogram integral intensity of the aorta images have been compared with the aortic cholesterol content (ACC) data (see Figure 9). Relative MRI as well as ACC data was calculated by regular normalization procedure, and an aorta with minimum cholesterol content (see aorta \# 7 in Table 1) was used as a reference. It was noted an apparent visual agreement between MRI data in Figure 9 and corresponding excised arterial segments in Figure 1. To obtain more detailed MRI data, a well-known calibration protocol can be employed with the use of a calibrated reference sample or staining. ${ }^{[8,10]}$

Linear regression analysis (see Figure 9) shows good correlation between MRI and cholesterol content in the thoracic aortas examined. Pearson correlation was calculated for statistical comparison of atherosclerotic lesions measurements obtained by MRI and the aortic cholesterol content. A significant correlation was found with Pearson correlation coefficient $r=0.95$.

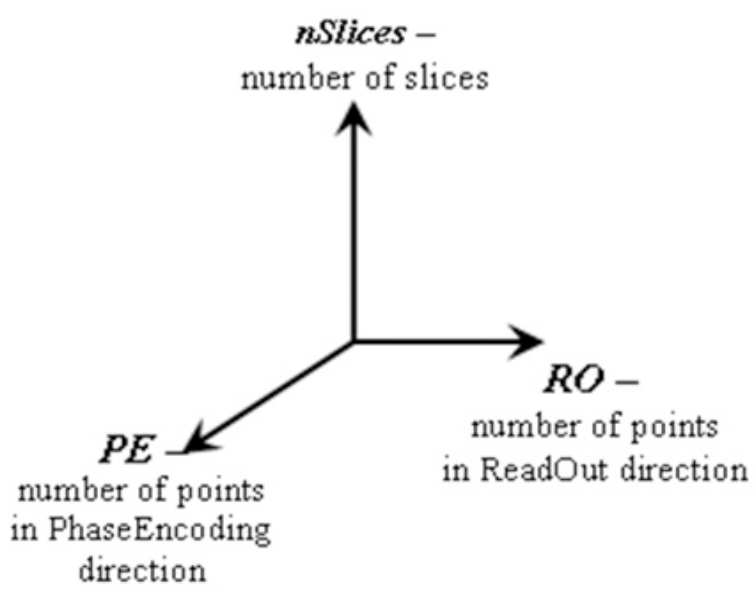

Figure 6. Coordinated system used in a slice compressing procedure (see step 4 in Figure 3)

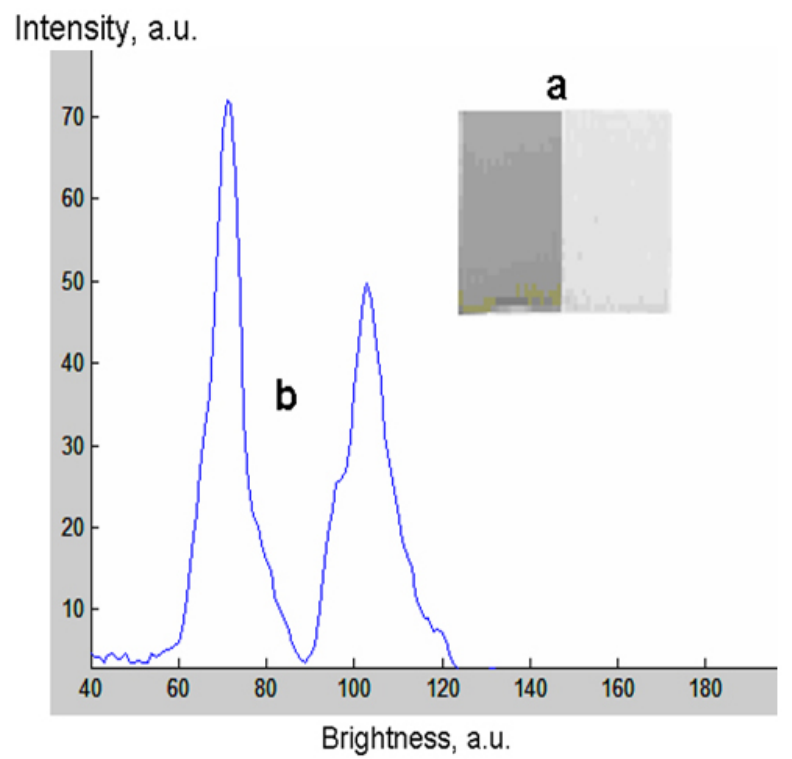

Figure 7. (a) 2D compressed MRI images of two phantoms with known and different proton concentrations and $\mathrm{T}_{2}$ relaxation times; (b) corresponding histograms

\section{Conclusions}

The developed CSH analysis has allowed obtaining reliable statistically averaged MRI data of the total atherosclerotic lesion of aorta without using any contrast reagents. The results show that with an appropriate set of imaging parameters a histogram integral intensity of compressed 2D aorta images can be employed as a quantitative distribution of atherosclerotic lesions. CSH method does not require high 
spatial or temporal MRI resolution providing statistically weighted results. Different MRI modalities (e.g. weighting, different imaging pulse sequences, fat suppression, etc.) can be employed to assist with the differentiation components of complex atherosclerotic lesions. A fast 2-D slice by slice acquisition can be used to decrease MRI timing session. The reported findings indicate significant correlations between results obtained with the cholesterol content of aorta and MRI lesion assessment, thus potentially allowing use of the non-invasive CSH-MRI for characterizing lesions in humans and animals with arterial disease. The developed fast postprocessing algorithm can be incorporated into MRI scanner software to meet minimal user-interaction requirements. The results suggest that the developed CSH-MRI analysis may thus be clinically useful, in view of the growing need to evaluate the progression and regression of atherosclerotic lesion of aorta without the requirement of invasive procedures.

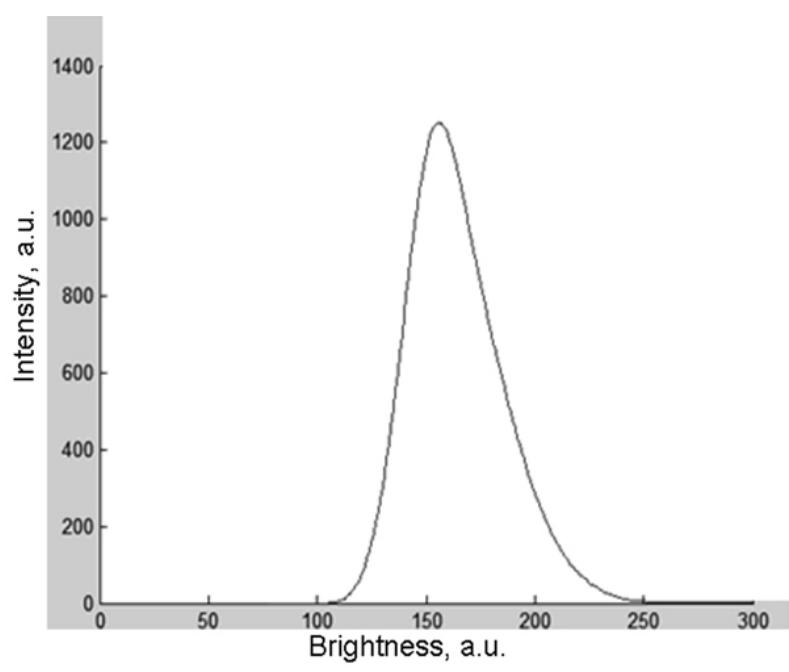

Figure 8. Final histogram integral intensity of the compressed 2D aorta images with a noise histogram subtracted (see steps 5-7 in Figure 3)

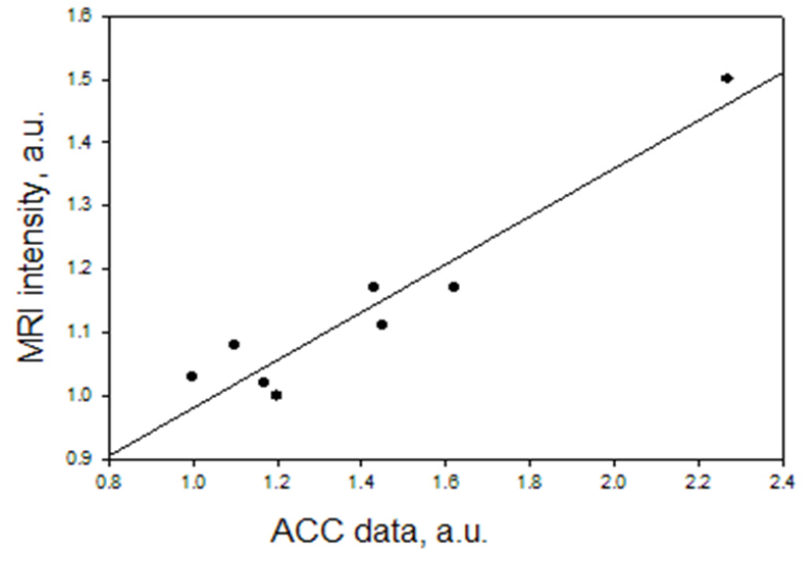

Figure 9. Linear regression analysis between MRI and aortic cholesterol contents $(r=0.95)$

Table 1. Total cholesterol contents of the thoracic aorta

\begin{tabular}{ll}
\hline Animal \# & Total cholesterol content $(\mathbf{m g} / \mathbf{g}$ wet tissue) \\
\hline 1 & 17.73 \\
2 & 22.18 \\
3 & 24.74 \\
4 & 18.31 \\
5 & 21.72 \\
6 & 18.13 \\
7 & 15.21 \\
8 & 34.56 \\
\hline
\end{tabular}

\section{ACKNOWLEDGEMENTS}

This research was supported in part by the Harlan E. Moore Heart Research Foundation. Resources of the Biomedical Imaging Center of the Beckman Institute for Advanced Science and Technology at the University of Illinois were used.

\section{REFERENCES}

[1] Ross R. Atherosclerosis - an inflammatory disease. New England Journal of Medicine. 1999; 340: 115-126. http://dx.doi.org/1 $0.1056 /$ NEJM199901143400208

[2] Moreno PR, Falk E, Palacios IF, et al. Macrophage infiltration in acute coronary syndromes: implications for plaque rupture. Circulation. 1994; 90: 775-8. http://dx.doi.org/10.1161/01.CIR. 90.2 .775

[3] Pignoli P, Tremoli E, Poli A, et al. Intimal plus medial thickness of the arterial wall: a direct measurement with ultrasound imaging. Circulation. 1986; 74: 1399-1406. http://dx.doi.org/10.1161 /01.CIR.74.6.1399

[4] Aqel RA, Zoghbi GJ, Baldwin SA, et al. Prevalence of renal artery stenosis in high-risk veterans refer to cardiac catheterization. Journal of Hypertension. 2003; 21: 1157-1162.

[5] Tearney GJ, Yabushita H, Houser SL, et al. Quantification of macrophage content in atherosclerotic plaque by optical coherence tomography. Circulation. 2003; 107: 113-119. http://dx.doi.o rg/10.1161/01.CIR.0000044384.41037.43

[6] Pearlman JD, Southern JF, Ackerman JL. Nuclear magnetic resonance microscopy of atheroma in human coronary arteries. Angiology. 1991; 42: 726-733. http://dx.doi.org/10.1177/00033 1979104200906

[7] Gold GE, Pauly JM, Glover GH, et al. Characterization of atherosclerosis with a $1.5 \mathrm{~T}$ imaging system. Journal of Magnetic Resonance Imaging. 1993; 3: 399-407. http://dx.doi.org/10.1002/jmr 


\section{i. 1880030216}

[8] Worthley SG, Helf G, Fuster V, et al. Serial In Vivo MRI Documents Arterial Remodeling in Experimental Atherosclerosis. Circulation. 2000; 101: 586-593. http://dx.doi.org/10.1161/01.CIR. 10 1.6 .586

[9] Helft G, Worthley SG, Fuster V, et al. Progression and regression of atherosclerotic lesions. Monitoring with serial noninvasive magnetic resonance imaging. Circulation. 2002; 105: 993-998. http://dx.doi.org/10.1161/hc0802.104325

[10] Skinner MP, Yuan C, Mitsumori L, et al. Serial magnetic resonance imaging of experimental atherosclerosis detects lesion fine structure, progression and complications in vivo. Nature Medicine. 1995; 1 : 69-73. http://dx.doi.org/10.1038/nm0195-69

[11] Helft G, Worthley SG, Fuster V, et al. Atherosclerotic aortic component quantification by noninvasive magnetic resonance imaging: an in vivo study in rabbits. Journal of American College of Cardiology. 2001; 37: 1149-1154. http://dx.doi.org/10.1016/S0735-1 $097(01) 01141-X$

[12] Dalager-Pedersen S, Falk E, Ringgaard S, et al. Effects of temperature and histopathologic preparation on the size and morphology of atherosclerotic carotid arteries as imaged by MRI. Journal of Magnetic Resonance Imaging. 1999; 10: 876885. http://dx.doi.org/10.1002/(SICI) 1522-2586(1999 11) $10: 5<876::$ AID-JMRI37>3.0.CO;2-T

[13] Folch L, Lees M, Sloane-Stanley GH. A simple method for the isolation and purification of total lipids from animal tissues. Journal of Biological Chemistry. 1957; 226: 497-509. Available from: http://www.jbc.org/content/226/1/497.full.pdf. PMid: 13428781.

[14] Kishida T, Nogami H, Ogawa H, et al. Hypocholesterolemic effect of high amylase cornstarch in rats is mediated by an enlarged bile acid pool and increased fecal bile acid excretion, not by cecal fermented products. Journal of Nutrition. 2002; 132: 2519-2524. Available from: http://jn.nutrition.org/content/132/9/2519. full.pdf+html. PMid: 12221203.

[15] Toussaint JF, Southern JF, Fuster V, et al. T2-weighted contrast for NMR characterization of human atherosclerosis. Arteriosclerosis Thrombosis Vascular Biology. 1995; 15: 1533-1542. Available from: www.ncbi.nlm.nih.gov/pubmed/7583524. PMid: 7583524.

[16] McConnell MV, Aikawa M, Maier SE, et al. MRI of Rabbit Atherosclerosis in Response to Dietary Cholesterol Lowering. Arteriosclerosis Thrombosis Vascular Biology. 1999; 19: 1956-1959. http://dx.doi.org/10.1161/01.ATV.19.8.1956

[17] Shinnar M, Fallon JT, Wehrli S, et al. The diagnostic accuracy of ex vivo MRI for human atherosclerotic plaque characterization. Arteriosclerosis Thrombosis Vascular Biology. 1999; 19: 2756-2761. http://dx.doi.org/10.1161/01.ATV.19.11.2756

[18] Worthley SG, Helft G, Fuster V, et al. High resolution ex vivo magnetic resonance imaging of in situ coronary and aortic atherosclerotic plaque in a porcine model. Atherosclerosis. 2000; 150: 321-329. http://dx.doi .org/10.1016/S0021-9150(99)00386-X

[19] Viereck J, Ruberg FL, Qiao Y, et al. MRI of Atherothrombosis Associated With Plaque Rupture. Arteriosclerosis Thrombosis Vascular Biology. 2005; 25: 240-45. http://dx.doi.org/10.1161/01. ATV.0000149673.00564.0a 